\title{
Multichannel Recorder for Low Frequency Signals: Application of Oscilloscope as Integrated Mobile Service for a Smartphone
}

\author{
Michal Kochlan, Samuel Zak, Michal Hodon, Juraj Micek, and Ondrej Karpis \\ University of Zilina, Univerzitna 8215/1, 01026 Zilina, Slovakia \\ Correspondence should be addressed to Michal Kochlan; michal.kochlan@fri.uniza.sk
}

Received 5 February 2016; Accepted 21 April 2016

Academic Editor: Ondrej Krejcar

Copyright ( 2016 Michal Kochlan et al. This is an open access article distributed under the Creative Commons Attribution License, which permits unrestricted use, distribution, and reproduction in any medium, provided the original work is properly cited.

\begin{abstract}
Data acquisition and processing are well known for some time. Many applications use powerful hardware to acquire, process, and visualize signal waveforms. But there are some applications that do not have to perform high resolution signal acquisition and process large amount of data, for example, low frequency applications of embedded design and applications for remote power grid monitoring. The paper describes special system for low frequency signal data sample acquisition, processing, and visualization implemented as a service on Android-based smart device. The service makes smart device functioning as an oscilloscope or arbitrary waveform generator which is accessible remotely through Bluetooth. The design respects low power consumption requirements, simplicity, and user friendliness in application design. Application scenario was implemented as wireless data acquisition system for power grid monitoring.
\end{abstract}

\section{Introduction}

Utilization of powerful hardware for signal data acquisition, processing, and visualization is advantageous only in some cases. Advancements in silicon industry, especially among the chip producers, cause still deeper and deeper demand for effective solutions. Such solutions can provide sufficient performance versus energy consumption ratio. Special applications, such as those low frequency applications of embedded systems, ask for these results. It is not effective to serve higher sampling rates in these cases, since higher frequencies are accompanied with stronger noise influence. Also computational claims and power consumptions are higher when, though reliable, robust systems are employed.

Our aim was to target the field of testing and measurement equipment where present solutions are mostly built on the high-power, high-performance, high-quality solutions with high resolutions, which are very universal, but also very expensive. We tried to investigate solution, which would be not so robust but could be also very universal providing sufficient quality and reliability for not so demanding measurements. Such kind of low-cost solution can be distributed to additional users, so measurement multiplications can be reached.
Selection of control unit plays the crucial role in the system architecture specification. Implementation of smart device, for example, smartphone, tablet, or watch, can serve the purpose of relatively powerful and reliable technology. Due to relatively massive spread of such devices among ordinary people, the financial issues can be neglected.

Present smart devices combine several modules in one device, sensory, communication, computation, and GUI, so they are predicted to be employed for different additional purposes, instead of nowadays traditional usage.

Our system was implemented as a multichannel recorder in application of an oscilloscope device designed to collect, display, and generate continuous arbitrary waveforms together with the smart device which serves the purpose of the visualization unit. Both devices communicate wirelessly via Bluetooth. This standard allows connecting almost any modern smart device (smartphone, tablet, laptop, etc.) since they all already incorporate Bluetooth connectivity. At the same time, the wireless communication provides galvanic isolation between the measured system (connected to oscilloscope) and visualization unit, smart device. The advantage of the wireless connectivity lies also in mobility of the user. Block schematic of the system in a measurement scheme is shown in Figure 1. 


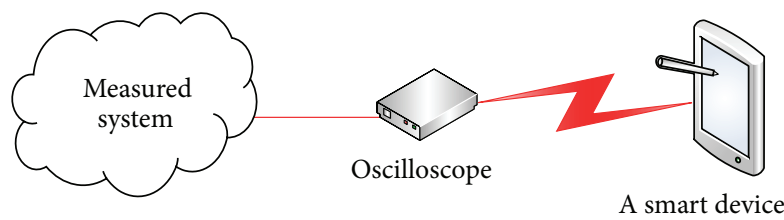

Figure 1: Block schematic of the system.

Different systems were already released on the market, including amateur solutions:

(i) Xminilab [1], a mixed signal oscilloscope based on ATXmega32 with 2 analog inputs and maximum sampling frequency 2 Msps.

(ii) Oscillophone [2], a visualization unit for signals ranging from $150 \mathrm{~Hz}$ to $15 \mathrm{kHz}$ along with an embedded signal generator output.

(iii) Xprotolab [3], a mixed signal oscilloscope with arbitrary waveform generator based on ATXmega microcontroller with 8-bit resolution and up to 2 Msps.

Professional solutions $[4,5]$ are capable of sampling at least in $100 \mathrm{MHz}$ frequency on each channel. These solutions are based on FPGA processing units.

Our aim was to target power metering application domain, where utilization of such system could be beneficial for more users. Such different attempts were already examined; for example, in [6-10], various wireless indoor power data acquisition systems were described. Several approaches and implementation schemes were utilized; however all mentioned solutions were implemented only as a specific application. System versatility was missing.

In [11-14], similar solutions to our design were described; however, they were implemented mostly for educational purposes. Disadvantages and advantages of WPANs utilizations for the purposes of remote metering were investigated in [1519]. Solutions compatible with the true definition of a portable oscilloscope can be found in [20-22].

\section{Technical Implementation}

The oscilloscope end device has eight analog differential input channels (IN1 to IN8) with programmable sensitivity. All the inputs are routed to the terminals. One analog output channel for arbitrary waveform generator function is routed to the terminal connector as well. The communication with the user visualization device, a smartphone or a tablet, is implemented using BTM112 Bluetooth module. In order to save the measured data a MicroSD card slot is implemented on-board.

Analog part of the device design is powered directly by a single cell Li-Ion battery with capacity of $680 \mathrm{mAh}$. This part consists of preprocessing circuitry made by a network of miniature, high-precision, low quiescent current operational amplifiers OPA2333. These OPAs can be supplied by voltage as low as $+1.8 \mathrm{~V}( \pm 0.9 \mathrm{~V})$ and up to $+5.5 \mathrm{~V}( \pm 2.75 \mathrm{~V})$. Regarding their optimized design for low-voltage, singlesupply operated applications, they represent ideal choice for oscilloscope design. The network of interface OPA2333 operational amplifiers is connected to the programmable gain operational amplifier PGA117. This programmable gain amplifier (PGA) can be set via an SPI interface. It has 10 analog inputs, from which 8 are used as analog inputs and two are used as reference lines. The scope gain of the PGA can be programmed from 1- up to 200 -fold of the input signal.

The digital part of the design is powered through the low-dropout, low-power linear regulator TPS71701. The main element of the digital part as well as the whole oscilloscope device is MSP430F6638 microcontroller. This device is ultra-low-power microcontroller consisting of several devices featuring different sets of peripherals targeted for various applications. The architecture, combined with five low-power modes, is optimized to achieve extended battery life in portable measurement applications. MSP430F6638 is a microcontroller configuration with a high-performance 12-bit analog-to-digital converter (ADC), comparator, two universal serial communication interfaces (USCI), USB 2.0, a hardware multiplier, and many more. Since the typical applications for this device include analog and digital sensor systems, digital motor control, remote controls, thermostats, digital timers, and hand-held meters, the selection of this device for the oscilloscope application was obvious. A block diagram of the oscilloscope implementation is presented in Figure 2.

User device parameters of the oscilloscope device are limited by used electronic components and modules:

(i) Transmission speed between the smart device and the oscilloscope device is limited to $460.8 \mathrm{kbps}$.

(ii) Communication distance of Bluetooth class 2 module, BTM112, is limited to $10 \mathrm{~m}$.

(iii) Sampling frequency of the oscilloscope device is up to $300 \mathrm{ksps}$ (using a single channel having other channels nonutilized).

(iv) Battery operating time is at least 8 hours in continuous data transmitting mode.

The described technical means allow the device to work in three basic modes. The user can choose one of the following operating modes:

(i) 8-channel oscilloscope;

(ii) 1-channel arbitrary waveform generator;

(iii) 1-8-channel data logger of the acquired samples.

It should be noted that the software as well as the hardware implementation of the proposed device is under continuous development.

In 8-channel oscilloscope mode (see Figure 3), the device is capable of displaying four channels at a time. The limitation of the input signal is affected by the input limits of the interface network of the operational amplifiers OPA2333. Also the number of the active channels plays significant role in the limitation of the input signal (frequency) properties. The key limitation represents the sampling frequency that is $300 \mathrm{ksps}$ at maximum setting (single channel utilization). In this mode, vertical range, amplitude scaling, can be adjusted in the steps 


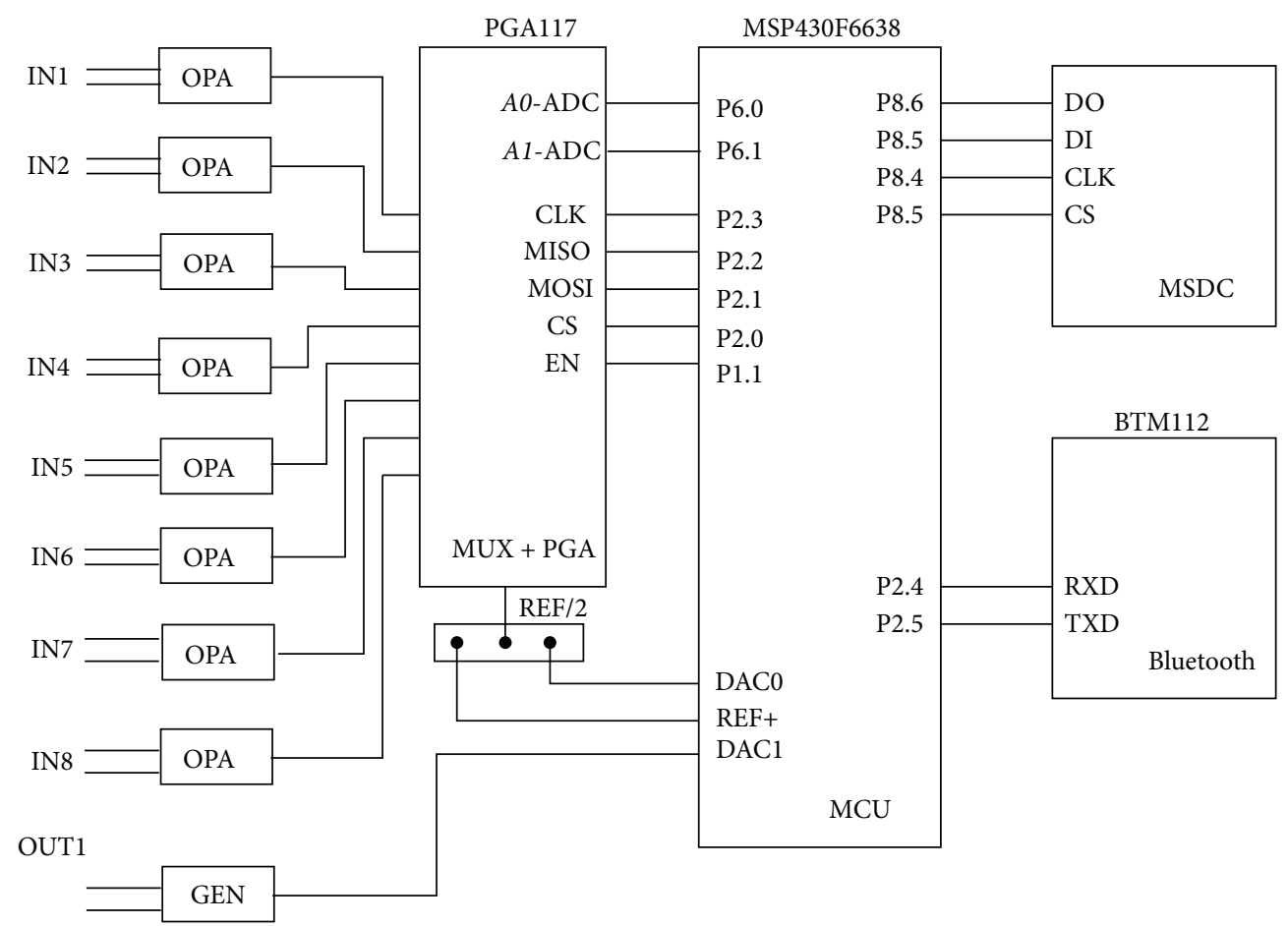

FIGURE 2: Block diagram of the oscilloscope device.

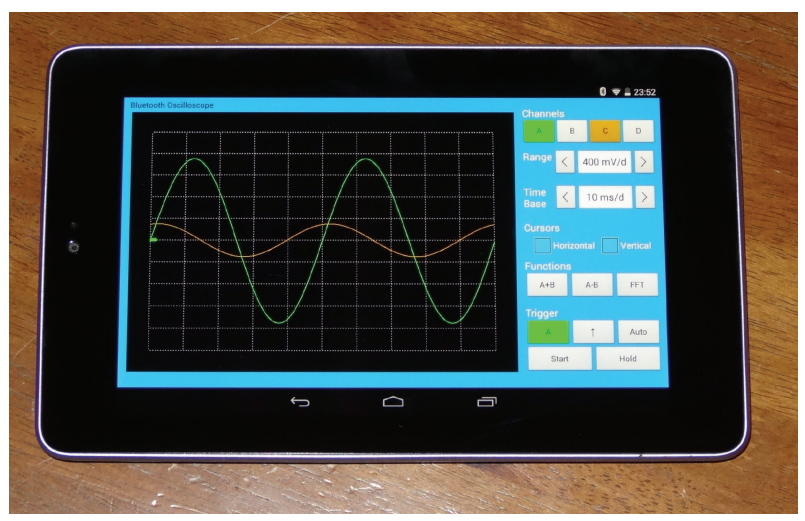

Figure 3: Oscilloscope mode screen.

of $10 \mathrm{mV}$ per segment. The time axis can be set to change in $10 \mathrm{~ms}$ per segment. Vertical and horizontal cursor can be enabled as well. Simple functions such as Fast Fourier Transform of the inspected signal, difference of the measured signals, and addition of two measured signals can be used by selecting proper button. Triggering function allows triggering the measurements on rising or falling edge. All the outputs can be inspected in real time or can be held steady until user intervention.

The 1-channel arbitrary waveform generator uses DAC converter of the main microcontroller MSP430F6638. The limits of the output voltage range are from $0 \mathrm{~V}$ up to AVCC that equals $3.3 \mathrm{~V}$. Time to change the settings FSR is in range $10-100 \mu \mathrm{s}$.

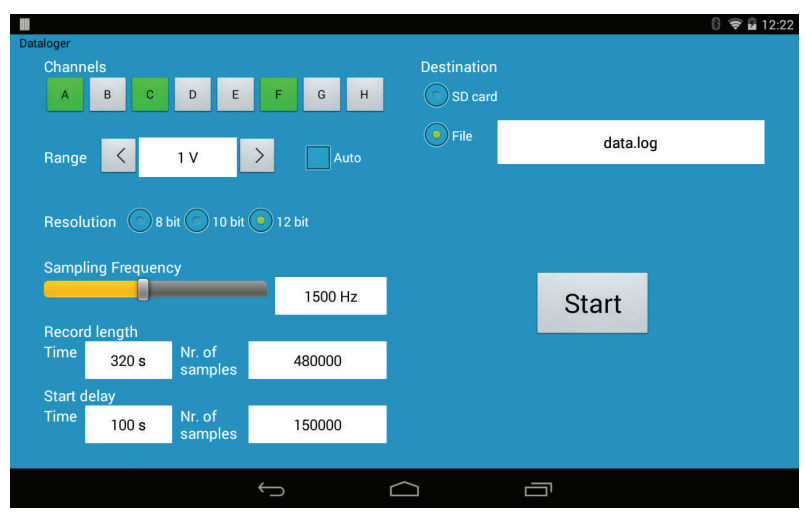

FIGURE 4: Datalogger mode screen.

Figure 4 shows the datalogger function. Measured values are being recorded into the embedded MicroSD card from the chosen channels 1 to 8 . User can select the output file as well. According to the selected sampling period (frequency) $100 \mathrm{~ms}$ up to $4 \mu \mathrm{s}$. As the number of channels of the sampled signals increases, the sampling period decreases with the number of the selected channels.

Within this mode, the channels designated by letters "A" to " $H$ " can be enabled for data logging. Resolution of the sampled values can be chosen from three options -8 bits, 10 bits, and 12 bits. The sampling frequency can be adjusted from $100 \mathrm{~Hz}$ up to $300 \mathrm{kHz}$. Obviously, the similar limitations apply for the datalogger mode as well as for the oscilloscope mode. These limitations relate to the capability of the datalogger to sample the values close to the upper limit $300 \mathrm{kHz}$ only in 
case a single channel is used. The more channels are utilized for datalogging, the lower the sampling frequency can be. For example, using all 8 channels, the maximum achievable sampling frequency per a single channel is $30 \mathrm{~Hz}$.

In testing application scenario, one channel measures the electric grid voltage on the voltage divider adapted for its input range. The second channel measures the voltage at the current transformer "AX-1000" from the manufacturer "Talema." Mains conductor passes through the transformer to the device connected to the outlet adapter. The voltage at the transformer is thus proportional to the current flowing through the device (current includes also its own consumption). The maximum operating current which can microcontroller converter measure is $10 \mathrm{~A}$. The output current flows through the power latching relay "JSL-D5N-K" by manufacturer "Fujitsu." The relay is used to control the output of the two coils, which are activated by a voltage of $5 \mathrm{~V}$. The maximum power that can load the component part was set by the manufacturer to $2000 \mathrm{~W}$ [23].

Outlet adapter electronics is powered from the switching power supply from "Myrra" producer. The switching power supply changes the AC mains to stabilized operating voltage $3.3 \mathrm{~V}$ with a maximum current consumption $750 \mathrm{~mA}$. Manufacturer guarantees that average efficiency is $65 \%$ for this switching power supply. We did not observe any malfunctioning during its working. The outlet adapter includes a charge pump TPS60401 by Texas Instruments. The charge pump is connected as a voltage inverter and serves as a power supply for the bistable relay [23].

Changing the voltage using the charge pump is advantageous for this application. The main reason is its high efficiency and low circuit complexity. Low output current may seem like a disadvantage because of the higher current necessary to change the relay status. Usage of the bistable relay however means that the control current in the coils may not stay for the conservation of the relay. Its switch requires only short pulse. Since the relay is the only component that uses a negative potential, it does not matter if it is this tension that fluctuates. To switch the relay is sufficient to have a negative voltage branch of a sufficiently large capacity of which short pulse will pass [23].

The solution is based on the MSP430F6638 microcontroller that performs the basic processing and communication with the smart device over the Bluetooth communication module. The analog part consists of the network of OPA2333 operational amplifiers that perform input signal preprocessing and programmable gain amplifier PGA117 that interfaces the network of the OPA2333 amplifiers with the microcontroller MSP430F6638. The digital part of the design is supplied by the low-dropout linear voltage regulator TPS71701.

\section{Use Case in Smart Grid Metering}

Oscilloscopes are used to observe the change of an electrical signal over time, such that voltage and time describe a shape which is continuously graphed against a calibrated scale. The observed waveform can be analyzed for such properties as amplitude, frequency, rise time, time interval, distortion, and others. Modern digital instruments may calculate and display these properties directly [22].

Many applications for data acquisition and processing use powerful hardware to acquire, process, and visualize signal waveforms. But there are applications that do not have to perform high resolution signal acquisition and process large amount of data, for example, low frequency applications of embedded design in education and applications for remote power grid monitoring. This contribution focuses on an idea of an oscilloscope as an integrated mobile service for remote power grid monitoring. This requires low frequency signal data sample acquisition, processing, and visualization on the smart device. The design of the described solution respects low power consumption requirements, simplicity, and user friendly control application design. The idea and core design of this solution served as a case study background for an unnamed private company in Slovakia for a portable and wireless data acquisition system for power grid monitoring and portable oscilloscope and arbitrary waveform generator device.

Smart grid metering is one of the recent developments in the area of electric power systems that aid the use of nonconventional sources of energy in parallel with the conventional sources [23].

By realizing the two-way communication between the utility and the smart meters in the houses, smart grid enables a time-of-use tariff to reduce peak load through incenting residents to adopt a more efficient usage of domestic appliances [24-27]. Moreover, this is beneficial for power grid technicians as well. The proposed solution aims to help to inspect power grid and electric meters.

The smart device oscilloscope is designed to collect and display (also generate) continuous arbitrary waveforms. Wireless communication is performed via Bluetooth module. This communication technology allows connecting almost any modern smart device (smartphone, tablet, laptop, etc.) since all of them incorporate Bluetooth connectivity. At the same time, the wireless communication provides galvanic isolation between the measured system and visualization unit, smart device.

The power grid monitor is a system for energy consumption monitoring in the power grid. It consists of a measuring device in the form of the (built-in) power grid adapter and the display device. The task of the power grid adapter is voltage and current measurement of the power grid. The measured data are sent wirelessly to visualization device. The power grid adapter consumes no more than $30 \mathrm{~mW}$ during the measurements. The outlet adapter is able to turn on and off the output, which allows controlling the remote devices or whole power grid branches. Moreover, switching can be automated on the time basis, power consumption, or the adapter temperature. This feature enables using the power grid adapter as an electronic fuse as well [21].

Application area of the developed device consists of remote power grid monitoring, embedded systems education, and measurement education. Core duties are evaluating congestion and grid stability, monitoring equipment health, energy theft prevention, and control strategies support. 


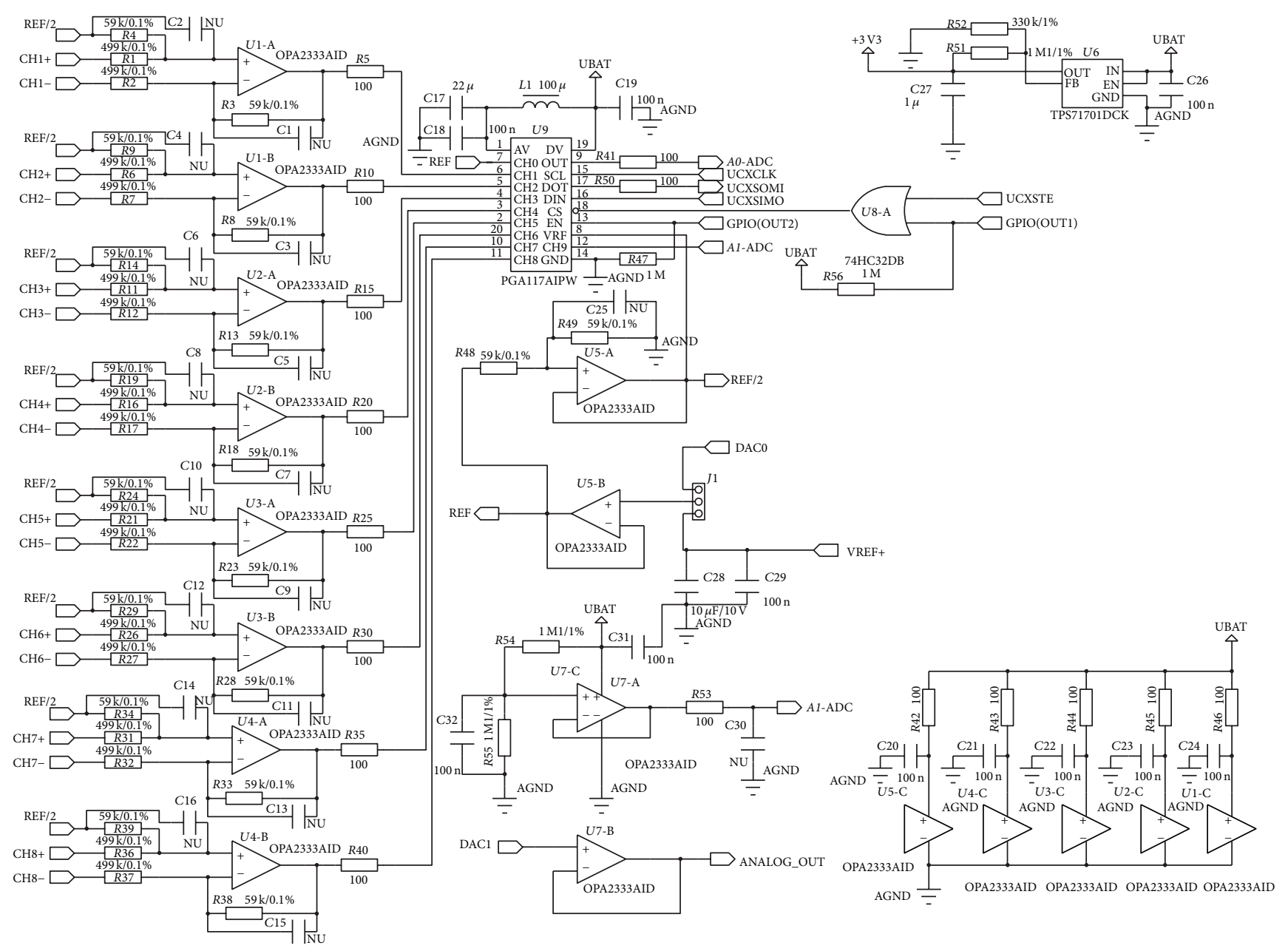

Figure 5: Control unit schematic.

Today, also electromagnetic signature measurement/analysis is important; time-of-use and real-time pricing tools gain popularity and advanced switches and cables.

The proposed device is suitable for smart buildings. It enables the power grid service workers to keep better maintenance, inspection, deviations, and noise in the power grid. Direct control of the power supply of devices is possible as well.

The idea and core design served as a case study background for an unnamed private company in Slovakia for a portable and wireless data acquisition system for power grid monitoring and portable oscilloscope and arbitrary waveform generator device.

Power flow control devices, such as the designed power grid adaptor, clamp onto existing transmission lines to control the flow of power within. Transmission lines enabled with such devices support greater use of (not only renewable) energy by providing more consistent, real-time control over how that energy is routed within the grid. This technology enables the grid to more effectively distribute the energy and possibly store intermittent energy from renewables for later use. Particular system schematics are shown in Figures 5 and 6.

\section{Conclusion}

The paper described a system of mobile oscilloscope which consists of several modules. The sensory device is used for analog data acquisition, the oscilloscope device for data processing, and the mobile device for data visualization of continuous waveforms. Wireless communication was implemented through Bluetooth standard. The wireless communication provides galvanic isolation between the measured system and visualization smart device. Simplicity of the design and ultra-low-power requirements are the key features of the system. The utilization of the proposed device can be found in diagnosis such as devices and systems that require isolation of the measurement device and visualization unit. The proposed device can be characterized by simple visualization layout, simple maintenance, low cost, and low power.

\section{Competing Interests}

The authors declare that they have no competing interests. 


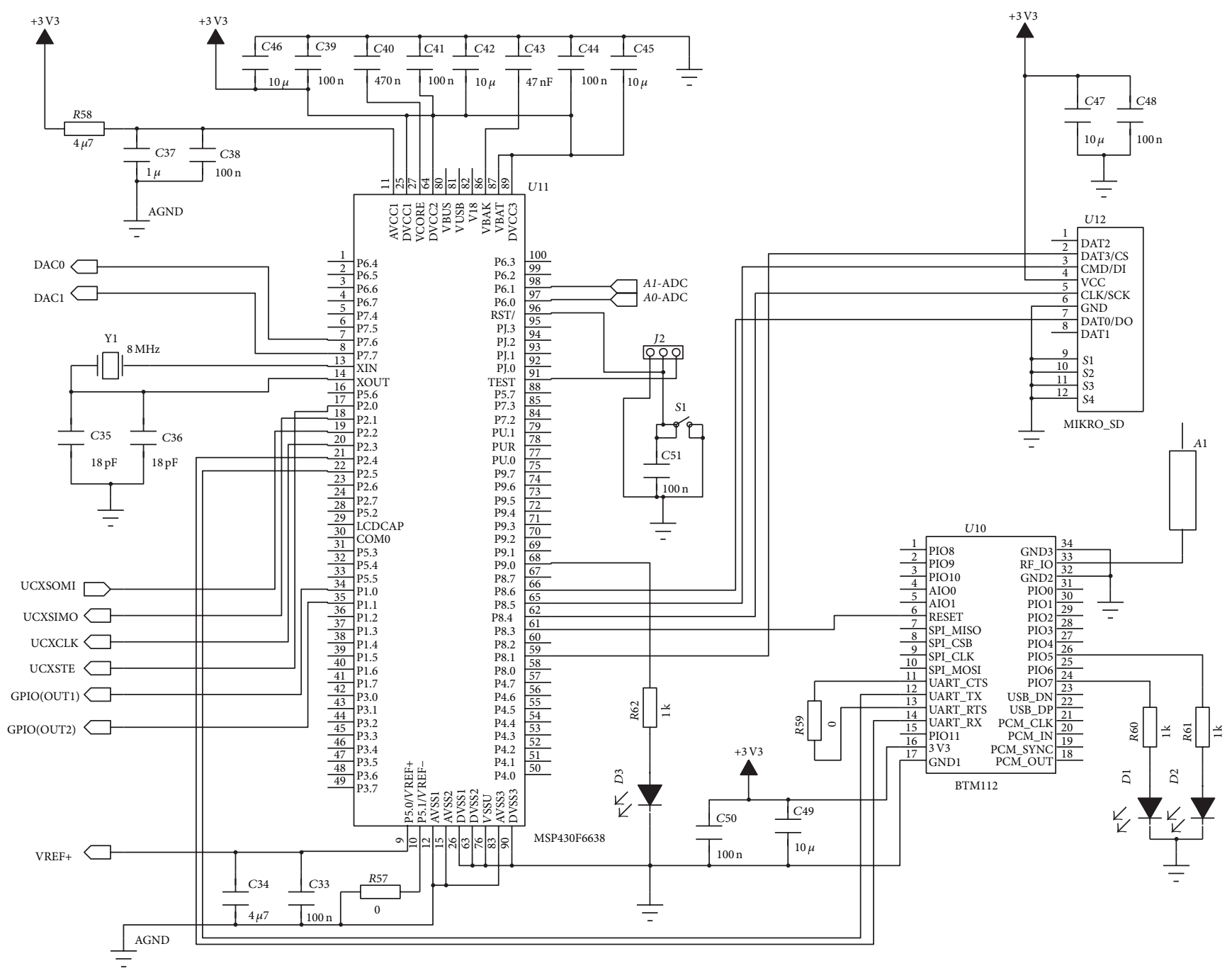

FIGURE 6: Control unit schematic.

\section{Acknowledgments}

The research is supported by the European Regional Development Fund and the Slovak state budget for the project "Modernization of the Research Infrastructure in Electrotechnic, Materials in Elektrotechnic, and Information Technologies", ITMS 26210120021.

\section{References}

[1] Xminilab Portable, http://www.gabotronics.com/oscilloscopes/ xminilab-portable-oscilloscope.htm.

[2] OscilloPhone: Use your Smartphone as an Oscilloscope/Signal Generator, http://www.instructables.com/id/OscilloPhone-Useyour-Smartphone-as-an-Oscilloscop/.

[3] Xprotolab, http://www.gabotronics.com/development-boards/ xmega-xprotolab.htm.

[4] SmartScope, https://www.lab-nation.com/.

[5] ELITEpro XC Power Meter, The Wireless Energy Datalogger from DENT Instruments, http://www.dentinstruments.com/ video-elitepro-sp-powermeter-communications-options.
[6] C.-H. Huang, T.-T. Hsien, and G.-J. Jong, "Indoor power meter combined wireless sensor network for smart grid application," in Proceedings of the 8th International Conference on Information Science and Digital Content Technology (ICIDT '12), vol. 2, pp. 336-339, Jeju Island, Republic of Korea, June 2012.

[7] W. A. Souza, F. P. Marafão, E. V. Liberado, I. S. Diniz, and P. J. A. Serni, "Power quality, smart meters and additional information from different power terms," IEEE Latin America Transactions, vol. 13, no. 1, pp. 158-165, 2015.

[8] X. Peng and Y. Xiao, "An embedded electric meter based on bluetooth data acquisition system," in Proceedings of the 2nd International Workshop on Education Technology and Computer Science (ETCS '10), pp. 667-670, Wuhan, China, March 2010.

[9] G. Romero and E. Inga, "State of art, MVNO for advanced metering infrastructure based on radio cognitive for channel allocation," IEEE Latin America Transactions, vol. 13, no. 9, pp. 2998-3004, 2015.

[10] J. Brenkus, V. Stopjakova, R. Zalusky, J. Mihalov, and L. Majer, "Power-efficient smart metering plug for intelligent households," in Proceedings of the 25th International Conference Radioelektronika (RADIOELEKTRONIKA '15), pp. 110-113, IEEE, Pardubice, Czech Republic, April 2015. 
[11] A. Wagh, Z. Dave, G. Singh, and V. Dange, "A low cost portable oscilloscope for educational platforms using a Programmable System on Chip," in Proceedings of the International Conference on Advances in Communication and Computing Technologies (ICACACT '14), pp. 1-4, Mumbai, India, August 2014.

[12] W. H. Siew, Y. Wang, and M. Faheem, "Wireless digital data acquisition system for EMI measurement in power substations," in Proceedings of the 17th International Zurich Symposium on Electromagnetic Compatibility (EMC-Zurich '06), pp. 288-291, Singapore, February-March 2006.

[13] R. Katona and D. Fodor, "Texas instruments MSP430 microcontroller based portable multi-purpose instrument for android platforms," in Proceedings of the 6th European Embedded Design in Education and Research Conference (EDERC '14), pp. 1-5, Milano, Italy, September 2014.

[14] Y. Lobsiger, G. Ortiz, D. Bortis, and J. W. Kolar, "Concept and experimental evaluation of a novel DC- $100 \mathrm{MHz}$ wireless oscilloscope," in Proceedings of the 7th International Power Electronics Conference (IPEC-Hiroshima 2014 - ECCE-ASIA), pp. 1309-1316, Hiroshima, Japan, May 2014.

[15] K. Nair, J. Kulkarni, M. Warde et al., "Optimizing power consumption in iot based wireless sensor networks using Bluetooth Low Energy," in Proceedings of the International Conference on Green Computing and Internet of Things (ICGCIoT '15), pp. 589593, Noida, India, October 2015.

[16] V. C. Gungor, B. Lu, and G. P. Hancke, "Opportunities and challenges of wireless sensor networks in smart grid," IEEE Transactions on Industrial Electronics, vol. 57, no. 10, pp. 35573564, 2010.

[17] G. P. Hancke Jr. and C. F. Mbuya, "Dynamic spectrum allocation for smart meter networks and WSNs in the presence of household consumer networks," in Proceedings of the IEEE 23rd International Symposium on Industrial Electronics (ISIE '14), pp. 1459-1462, IEEE, Istanbul, Turkey, June 2014.

[18] M. Erol-Kantarci and H. T. Mouftah, "Wireless sensor networks for cost-efficient residential energy management in the smart grid," IEEE Transactions on Smart Grid, vol. 2, no. 2, pp. 314325, 2011.

[19] J. Puchyova, M. Kochlan, and M. Hodon, "Development of special smartphone-based body area network: energy requirements," in Proceedings of the IEEE Federated Conference on Computer Science and Information Systems (FedCSIS '13), pp. 895-900, Kraków, Poland, September 2013.

[20] P. Sachin and A. D. Bhoi, "Portable measurement system powered by android," International Journal of Software \& Hardware Research in Engineering, vol. 2, no. 11, pp. 1-4, 2014.

[21] B. D. Hatwar and C. Amol, "Wireless oscilloscope powered by android," International Journal of Emerging Technology and Advanced Engineering, vol. 4, no. 7, 2014.

[22] J. Jiang, H. Qu, and S. L. Tian, "Study on the smart handheld wireless oscilloscope," Journal of Applied Mechanics and Materials, vol. 416-417, pp. 1325-1330, 2013.

[23] M. Hodoň, S. Žák, M. Kopkáš, P. Ševčík, and M. Húdik, "Application of WSN for smart power metering to avoid cheating on electric power consumption at places with shared power sources," in Proceedings of the Federated Conference on Computer Science and Information Systems (ACSIS '15), M. Ganzha, L. Maciaszek, and M. Paprzycki, Eds., vol. 5, pp. 12151221, September 2015.

[24] N. Kularatna, Fundamentals of Oscilloscopes, Digital and Analogue Instrumentation: Testing and Measurement, Institution of Engineering and Technology, 2003.
[25] S. Massoud Amin and B. Wollenberg, "Toward a smart grid: power delivery for the 21st century," IEEE Power and Energy Magazine, vol. 3, no. 5, pp. 34-41, 2005.

[26] R. V. P. Yerra, A. K. Bharathi, P. Rajalakshmi, and U. B. Desai, "WSN based power monitoring in smart grids," in Proceedings of the 7th International Conference on Intelligent Sensors, Sensor Networks and Information Processing (ISSNIP '11), pp. 401-406, Adelaide, Australia, December 2011.

[27] V. C. Gungor, D. Sahin, T. Kocak et al., "Smart grid technologies: communication technologies and standards," IEEE Transactions on Industrial Informatics, vol. 7, no. 4, pp. 529-539, 2011. 

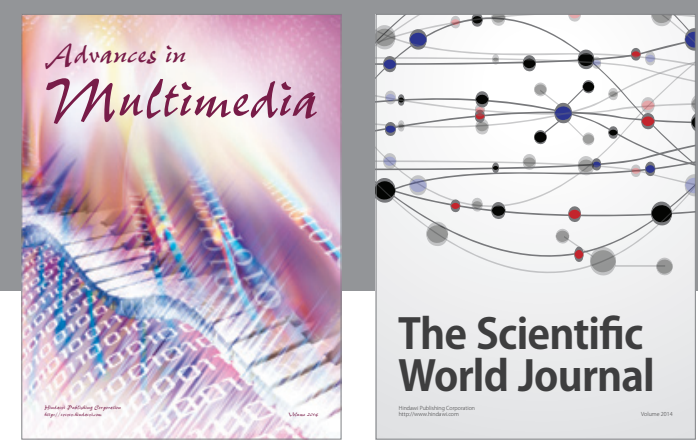

The Scientific World Journal
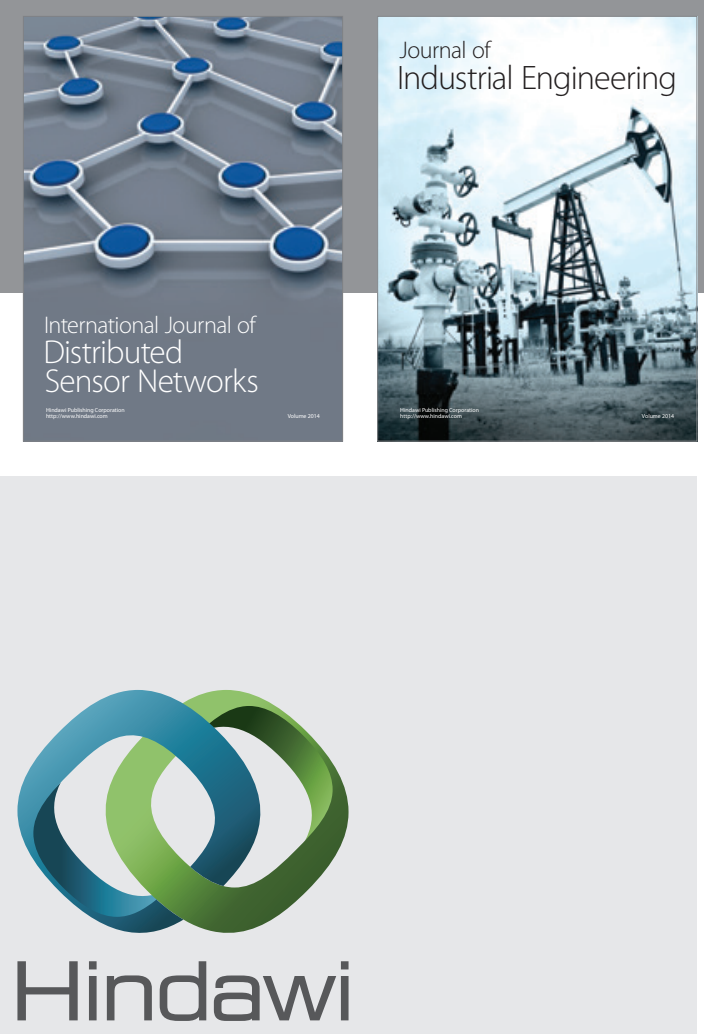

Submit your manuscripts at

http://www.hindawi.com

\section{Computer Networks} and Communications
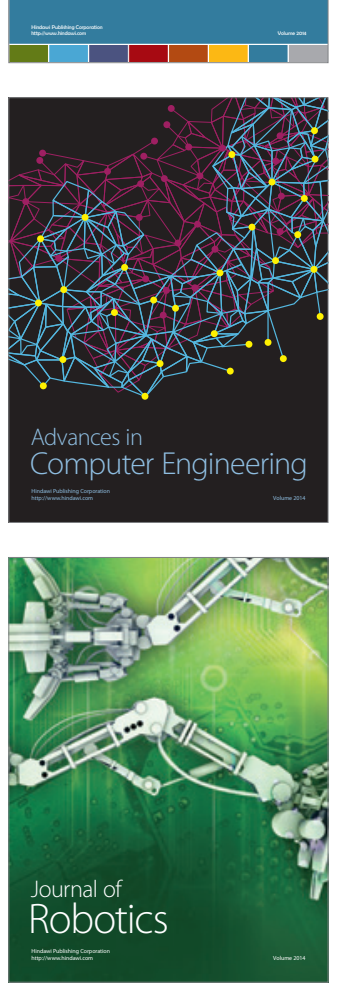
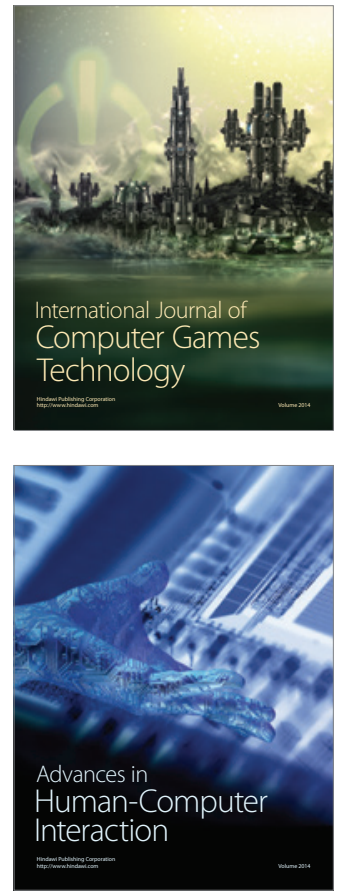
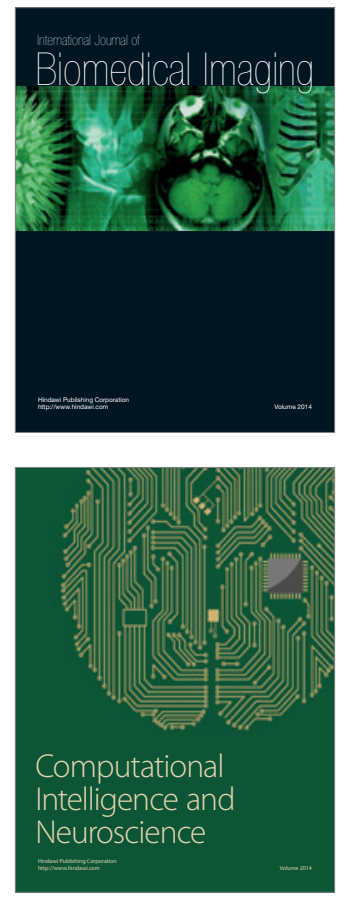
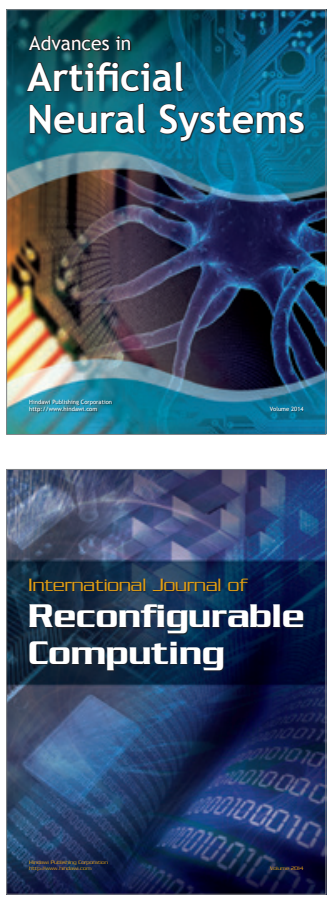
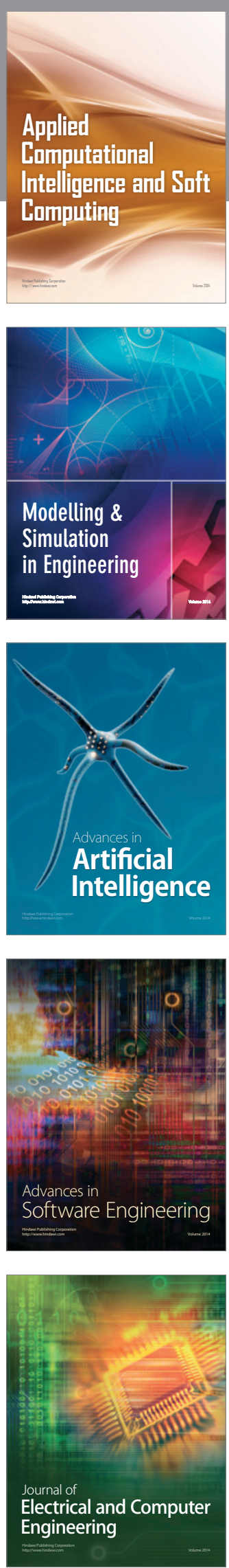